\title{
CREDIBILIDAD DE LOS MEDIOS DE COMUNICACIÓN: ANÁLISIS DE LA PRENSA DIARIA DESDE EL COMPORTAMIENTO DEL CONSUMIDOR
}

\author{
Cristina Calvo-Porral, Valentín-Alejandro Martínez-Fernández y Óscar \\ Juanatey-Boga
}

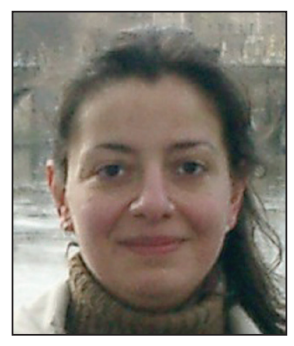

Cristina Calvo-Porral es licenciada en administración y dirección de Empresas por el lcade de Madrid (Universidad Pontificia de Comillas), y licenciada en investigación y técnicas de mercado por el mismo centro. Es doctora en economía por la Universidad de A Coruña y cuenta con numerosas publicaciones en el área de marketing. Su carrera profesional se ha desarrollado durante más de diez años en la industria de la moda, como responsable de Expansión Internacional de Carolina Herrera y Purificación García. Actualmente es profesora del Área de Comercialización e Investigación de Mercados de Facultad de Economía y Empresa de la Universidad de A Coruña. http://orcid.org/0000-0002-5470-0729
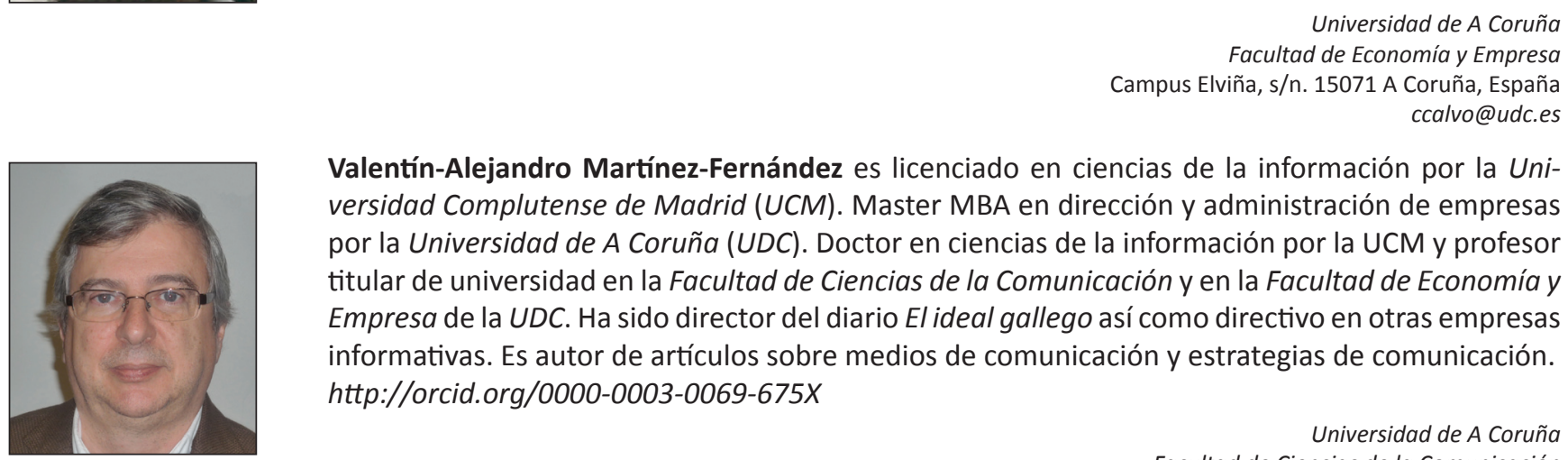

Valentín-Alejandro Martínez-Fernández es licenciado en ciencias de la información por la Universidad Complutense de Madrid (UCM). Master MBA en dirección y administración de empresas por la Universidad de A Coruña (UDC). Doctor en ciencias de la información por la UCM y profesor titular de universidad en la Facultad de Ciencias de la Comunicación y en la Facultad de Economía y Empresa de la UDC. Ha sido director del diario El ideal gallego así como directivo en otras empresas informativas. Es autor de artículos sobre medios de comunicación y estrategias de comunicación. http://orcid.org/0000-0003-0069-675X

Universidad de A Coruña Facultad de Ciencias de la Comunicación Campus de Elviña, s/n. 15071 A Coruña, España valejand@udc.es

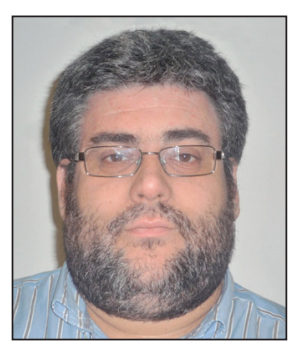

Óscar Juanatey-Boga es licenciado y doctor en ciencias económicas y empresariales por la Universidad de A Coruña (UDC). Master MBA en dirección y administración de empresas, master en dirección comercial y marketing, y master en comunicación empresarial por la UDC. Profesor del Área de Comercialización e Investigación de Mercados en la Facultad de Economía y Empresa de la misma universidad, es autor de artículos sobre medios de comunicación y estrategias de comunicación.

http://orcid.org/0000-0001-5226-2748
Universidad de A Coruña Facultad de Economía y Empresa Campus Elviña, s/n. 15071 A Coruña, España oscarjb@udc.es

\section{Resumen}

Se analiza la credibilidad de los medios de comunicación, y en concreto de los diarios generalistas. Mediante el empleo de un modelo de ecuaciones estructurales, y con una muestra de 454 cuestionarios sobre los diarios El país, El mundo, Abc, La razón y La voz de Galicia, se estudian los antecedentes y consecuencias de su credibilidad. Los resultados muestran la importante influencia de la calidad percibida, la imagen y la notoriedad del medio en la actitud de los consumidores, así como la influencia de la actitud sobre la credibilidad de que goza el medio. Los resultados conllevan interesantes implicaciones para los gestores de medios de comunicación, que deben priorizar la imagen y notoriedad del medio, así como la calidad que perciben los consumidores de información en un entorno de competencia creciente.

\section{Palabras clave}

Credibilidad, Actitud, Medios de comunicación, Prensa escrita, Diarios, Consumidores, Usuarios, Estudios de usuarios, Cuestionarios.

\section{Title: Media credibility: analysis of the daily press based on consumer behaviour}




\begin{abstract}
We analysed mass media credibility, focusing particularly on generalist journals. We carried out structural equation modeling, based on a sample comprised of 454 questionnaires relative to the newspapers El país, El mundo, Abc, La razón and La voz de Galicia, in order to analyze the antecedents and consequences of credibility. The results obtained show the important influence of perceived quality, media image and awareness on consumers' attitudes, along with the influence of attitude on media credibility. Our findings provide useful insights for mass media managers that may enhance media image and awareness, along with consumers' perceptions of quality, in an increasingly competitive environment..
\end{abstract}

\title{
Keywords
}

Credibility, Attitude, Mass media, Newspapers, Consumers, Users, User studies, Questionnaires.

Calvo-Porral, Cristina; Martínez-Fernández, Valentín-Alejandro; Juanatey-Boga, Óscar (2014). “Credibilidad de los medios de comunicación: análisis de la prensa diaria desde el comportamiento del consumidor". El profesional de la información, marzo-abril, v. 23, n. 3, pp. 300-309.

http://dx.doi.org/10.3145/epi.2014.may.10

\section{Introducción}

En las dos últimas décadas se advierte una gran proliferación de nuevos medios de comunicación social, así como una gran fragmentación de las audiencias, lo que ha cambiado significativamente el escenario de la industria de la comunicación en España (Chan-Olmsted; Yung-Wook, 2001). Se trata de un entorno altamente competitivo, en el cual la gestión de marca es para los medios una estrategia necesaria para crear y establecer su credibilidad (Vila-López; Küster-Boluda; Bigné, 2013).

Muchos consumidores no pueden juzgar si una información es verdadera o no, o si está manipulada o no, de modo que han de confiar en el medio de comunicación. La credibilidad se convierte así en uno de los criterios esenciales en la selección de un medio, lo que provoca que los poco creíbles sean rechazados o no tengan incidencia en la conformación de opinión. Que la credibilidad influya en el comportamiento del consumidor y sea un factor decisivo en el éxito periodístico y comercial de un medio de comunicación (Schweiger, 2000) lleva a plantearnos cuáles son sus variables determinantes, el proceso para evaluarla, y las características del medio que influyen en su valoración.

Hay pocos trabajos sobre credibilidad de los medios de comunicación desde la perspectiva del consumidor de información (Fombrun; Van-Riel, 2004; Rathnayake, 2008; Walsh et al., 2009). Y en este contexto se plantea el presente trabajo, con el objetivo de analizar los antecedentes y las consecuencias de la credibilidad de los medios de comunicación -más específicamente de los diarios generalistas de referencia-, desde la perspectiva del consumidor. Para ello se ha diseñado la investigación tomando como referencia los diarios El país, El mundo, Abc, La razón y La voz de Galicia.

\section{Revision de la literatura}

\subsection{Concepto de credibilidad de los medios}

La credibilidad es un concepto multidimensional (Gaziano; McGrath, 1986) y ha sido analizado tanto por la psicología social como por las ciencias de la comunicación. Si bien para la primera, la credibilidad es una variable subjetiva, defini- da por la percepción del receptor de la información (Tseng; Fogg, 1999), las ciencias de la comunicación señalan el carácter objetivo de la credibilidad, definida como el grado o nivel de precisión y exactitud de una información (Greer, 2003; Flanagin; Metzger, 2000). Hovland, Janis y Kelley (1953) propusieron el concepto de credibilidad de mayor influencia en la literatura (Erdem; Swait, 2004; Trimble; Rifon, 2006), señalando como sus dos componentes a la experiencia y la confianza. Definieron la credibilidad de una marca o producto, en este caso de un medio de comunicación, como la percepción del consumidor de su veracidad, confianza, buena voluntad y también de su experiencia.

\section{La credibilidad es un criterio esencial en la selección de un medio, lo que provoca que los poco creíbles sean rechazados o no tengan incidencia en la conformación de opinión}

La credibilidad del medio de comunicación se refiere al efecto que sus características concretas tienen sobre la credibilidad de sus mensajes, e igualmente a la carencia de un interés particular a la hora de transmitir información (Metzger et al., 2003). De acuerdo con Wathen y Burkell (2002), un medio de comunicación posee credibilidad si su contenido y sus mensajes son considerados válidos y de confianza. Por tanto su credibilidad se refiere a su buen hacer en términos de confiabilidad desde el punto de vista de los consumidores (Newell; Goldsmith, 2001). Autores como Nozato (2002) o Flanagin y Metzger (2007) definen los factores que permiten evaluar la credibilidad de un medio y entre ellos destacan experiencia, objetividad, dinamismo, equidad, ausencia de sesgo, integridad y exactitud, e igualmente fiabilidad y reputación.

En el caso concreto de la prensa, Hovland, Janis y Kelley (1953) identificaron dos factores principales de la credibilidad -confianza y experiencia-, además de señalar una serie de dimensiones subyacentes como son: la cualificación del medio, su dinamismo, su conocimiento y competencia, su 
precisión e imparcialidad. Finalmente, Gaziano y McGrath (1986) indican que la credibilidad de los diarios está formada por la información fiable y veraz, la ausencia de sesgo, la separación clara de información y opinión, la independencia de los intereses y medios que financian el diario, así como ofrecer la información de forma completa, mostrando los hechos tal y como son -contando la historia completa-.

La credibilidad de los medios de comunicación es similar a la de las marcas de productos o servicios, dado que es muy sencillo para el consumidor atribuir credibilidad a la marca de un medio reconocido (Schweiger, 2000). El consumidor de información es quien determina, en su rol de consumidor de noticias, la credibilidad de un medio. De esta manera, Liu (2004) define la valoración de la credibilidad de un medio como el proceso cognitivo mediante el que la información es filtrada y seleccionada por el consumidor; proceso en el cual las creencias del individuo, así como la actualidad, veracidad y calidad de la información tienen un impacto positivo. Por consiguiente, numerosos autores afirman que los consumidores tienden a escoger aquellos medios que perciben como más creíbles (Taylor, 1986; Rieh; Danielson, 2007).

La credibilidad de un medio de comunicación la determina el receptor, esto es, el consumidor de información

\section{Comportamiento del consumidor de información}

La Teoría del procesamiento de la información (Sughan, 1980) afirma que los consumidores toman decisiones en un contexto de riesgo e incertidumbre, y que desean reducir el coste asociado a sus decisiones de consumo. En este contexto se justifica que los consumidores escojan marcas (cabeceras) que gozan de credibilidad, para simplificar su proceso de compra y reducir el riesgo a equivocarse (Erdem; Swait, 1998). En el consumo de información, el consumidor tiene dos opciones: optar por creer lo que dicen los medios, sabiendo que tienen cierto sesgo informativo, en ocasiones neutralizado por la identificación con la línea editorial, o bien adoptar una postura crítica respecto a los mismos. Sin embargo, son pocos los consumidores que invierten tiempo en la formación de una opinión crítica, y por tanto tienden a asumir como cierto y creíble aquello que los medios de comunicación publican (Piñeros-Piza et al., 2011). Por tanto las creencias de los consumidores y su percepción, son variables que influyen en la credibilidad otorgada a un medio de comunicación (Blackwell; Engel; Miniard, 2002).

De acuerdo a lo señalado, la credibilidad de un medio de comunicación la determina el receptor, esto es, el consumidor de información. Por ello el presente trabajo se ha planteado desde el enfoque del consumidor, con el objetivo de analizar los antecedentes -calidad percibida, conciencia social e imagen y notoriedad- y las consecuencias de la credibilidad de los medios de comunicación -fidelidad y aceptación de nuevos productos-, para un tipo determinado de medio como son los diarios generalistas de referencia.

\subsection{Antecedentes y consecuencias de la credibilidad de los diarios}

\section{Calidad percibida}

Se entiende como calidad percibida de un medio de comunicación a la capacidad del medio para atender a una audiencia diversa, ofrecer contenidos relevantes y de actualidad, promover valores, generar conciencia y espíritu crítico, respetar las leyes y la cultura, o estimular la creatividad (Webster, 1986). Siguiendo a Wang y Soergel (1998), puede definirse como la valoración de la bondad de un medio en términos de calidad periodística. Por otro lado se relaciona con la respuesta subjetiva del consumidor hacia las características de un producto o servicio, y de esta manera, referida a un medio, se puede definir como el criterio del consumidor en cuanto a la excelencia y la veracidad del mismo (Taylor, 1986).

En este sentido Liu (2004) estima que la calidad percibida del medio de comunicación tiene un impacto positivo en su credibilidad. De acuerdo con todo ello, se plantea la siguiente hipótesis:

$H_{0}$ : La calidad percibida del diario tiene una influencia positiva en la actitud hacia el mismo

\section{Conciencia social}

En la línea de Hovland, Janis y Kelley (1953) y la de Gaziano y McGrath (1986), puede considerarse que la conciencia social incluye factores como el comportamiento ético y moral del medio en el tratamiento de la información, su compromiso con el interés público, así como la consideración de la opinión y punto de vista de los lectores. Además, estudios previos demuestran una relación positiva entre la conciencia social del medio y la actitud mostrada por los consumidores (Gaziano; McGrath, 1986). Consecuentemente se plantea la siguiente hipótesis:

$H_{1}$ : La conciencia social del diario tiene una influencia positiva en la actitud hacia el mismo

\section{Imagen y notoriedad}

Los consumidores asocian la credibilidad de un medio con su imagen general (Haley, 1996), al entender por tal la imagen efectiva que tienen de un medio concreto y que va más allá de la información que proporciona (Boulding, 1969). De esta manera la imagen, las asociaciones y el simbolismo asociado a un medio de comunicación contribuyen a su credibilidad e influyen en la actitud de los consumidores, dado que transmiten información sobre el mismo (Gotsi; Wilson, 2001). La imagen incluye la estética del contenido y la información emitida por el medio y las acciones llevadas a cabo por cualquier área de la empresa de comunicación (Carrillo; Castillo-Díaz; Tato-Jiménez, 2008). Numerosos autores afirman que las imágenes y asociaciones influyen en la credibilidad percibida del medio, y que los consumidores toman decisiones sobre su credibilidad basándose en asociaciones, imágenes o señales (Rieh; Danielson, 2007). Por otro lado diversos autores coinciden en que la notoriedad de un medio de comunicación, su familiaridad para el consumidor o su capacidad para reconocerlo y distinguirlo, también influye en la actitud hacia el mismo (Haley, 1996). De ahí que se 
proponga la siguiente hipótesis:

$H_{2}$ : La imagen y notoriedad del diario tiene una influencia positiva en la actitud hacia el mismo

\section{Actitud}

Estudios realizados en diferentes sectores e industrias, prueban la existencia de una relación positiva y significativa entre la actitud del consumidor, su credibilidad percibida y su comportamiento (Goldsmith; Lafferty; Newell, 2000; Lafferty; Goldsmith, 2004). La credibilidad es juicio subjetivo respecto a los atributos de un servicio o producto, y de-

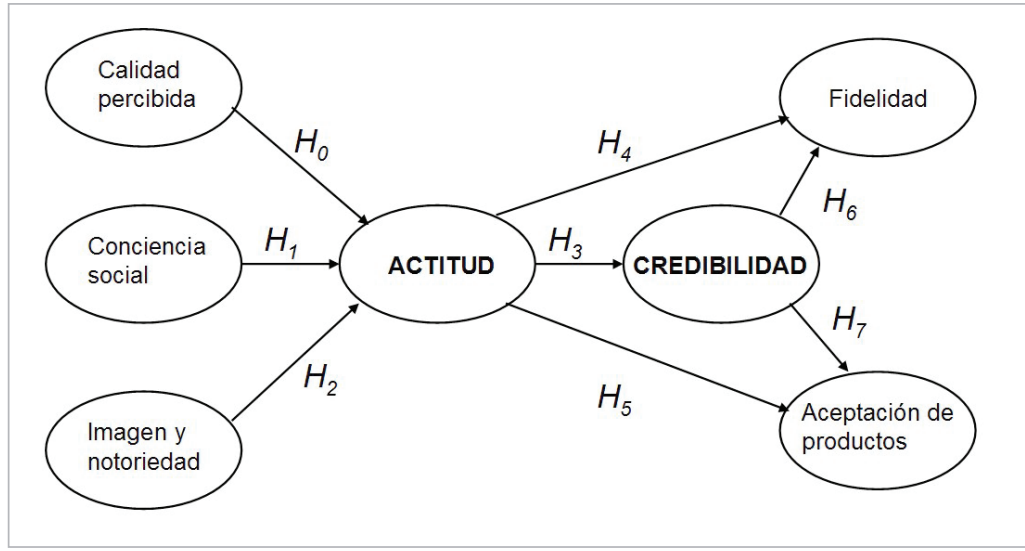

Figura 1. Modelo inicial propuesto pende de la actitud favorable o desfavorable hacia éstos (Piñeros-Piza et al., 2011). Consecuentemente la credibilidad de los medios de comunicación dependerá de la percepción que el consumidor tiene de una serie de variables -experiencia, confianza...-, así como de su actitud hacia el medio de comunicación en sí mismo (Fogg, 2003). Siguiendo a Meyer (1998), hay una clara correlación entre la credibilidad de los diarios y la actitud de la audiencia. Ello lleva a plantear la siguiente hipótesis:

$\mathrm{H}_{3}$ : La actitud hacia el diario tiene una influencia positiva en su credibilidad

\section{Fidelidad}

Puede definirse como el resultado de un proceso por el cual el consumidor adquiere asociaciones y percepciones positivas sobre un producto o marca, y que finalmente generará un compromiso por parte del consumidor (Dick; Basu, 1994). De esta manera, la fidelidad refleja el grado de satisfacción del consumidor con un producto o servicio (Aaker, 1991). Estudios previos vinculan la actitud del consumidor con su comportamiento (Mueller, 1957; Woo; Cho; Kwon, 2008); y según Rust y Donthu (1988), existe una relación positiva entre la actitud del consumidor hacia un medio de comunicación y su fidelidad hacia el mismo.

La credibilidad del medio de comunicación -su confiabilidad, su honestidad y experiencia- influirá también en el comportamiento del consumidor, y concretamente en la fidelidad y la aceptación de nuevos productos. Estudios previos señalan que los consumidores tienden a escoger aquellos medios que perciben como más creíbles (Rieh; Danielson, 2007). Finalmente, diversos autores han demostrado la relación entre la credibilidad del medio de comunicación y un incremento de consumo del mismo (Van-Weezel, 2006); así como de la fidelidad de la audiencia (Meyer, 2004). Por ello, presentamos las siguientes hipótesis:

$\mathrm{H}_{4}$ : La actitud hacia el diario tiene una influencia positiva en la fidelidad hacia el mismo

$H_{6}$ : La credibilidad del diario tiene una influencia positiva en la fidelidad hacia el mismo

\section{Aceptación de nuevos productos}

Una de las consecuencias que tienen en el comportamiento del consumidor la actitud y la credibilidad, es su predisposición hacia nuevos productos del medio. Diversos traba-

jos han demostrado la relación existente entre la credibilidad del medio y la aceptación hacia los nuevos productos que éste introduce (Bigné-Alcañiz, 1992; Amos; Holmes; Strutton, 2008). Así, se proponen las siguientes hipótesis:

$H_{5}$ : La actitud hacia el diario tiene una influencia positiva en la aceptación de nuevos formatos

$H_{7}$ : La credibilidad del diario tiene una influencia positiva en la aceptación de nuevos formatos

\section{Metodología}

\subsection{Selección de la muestra}

Se ha tenido en cuenta únicamente el canal o medio de información, en cuanto a variables de credibilidad, y se ha seleccionado exclusivamente prensa en soporte papel, prescindiendo de la digital (Pornpitakpan, 2004). Se siguieron dos criterios para la selección de diarios:

- tener amplia notoriedad y difusión; es decir, ser conocidos por la mayoría de los lectores y tener tirada y distribución nacional;

- presentar marcadas líneas editoriales.

Así, se realizó un primer estudio exploratorio para conocer los diarios de referencia en España, y conforme a estos resultados, se seleccionaron El país, El mundo, Abc, y La razón. También se decidió incluir a La voz de Galicia como cabecera representativa de los diarios de difusión autonómica. El método de muestreo fue aleatorio, enviando a cada participante un cuestionario correspondiente a uno de los diarios seleccionados. Los cuestionarios fueron enviados a personas residentes en el territorio español.

El método de recogida de información fue el cuestionario electrónico auto-administrado que agrupaba las distintas variables objeto de estudio mediante escalas clásicas tipo Likert de 5 puntos (siendo 1 totalmente en desacuerdo y 5 totalmente de acuerdo). Además se incluyeron variables socio demográficas como género, edad, nivel de renta o de estudios. El 44,5\% de los participantes eran hombres, frente a un 55,5\% de mujeres. El 19,48\% tenía estudios primarios, mientras, el $47,09 \%$ poseía un título universitario, un $27,71 \%$ estudios secundarios y un $5,72 \%$ de formación profesional. Por último, y en relación al nivel de renta, un 35,17\% ganaba entre 6.000 y 18.000 euros al año, un 36,48\% entre 18.000 y 30.000 , y un $16,5 \%$ declaraba unos ingresos superiores a los 
30.000 euros por año. El trabajo de campo se llevó a cabo durante los meses de marzo y abril de 2013 y se obtuvieron 454 cuestionarios válidos: El país (123), El mundo (88), Abc (80), La razón (65) y La voz de Galicia (98). Por último, el error muestral fue del $4,7 \%$, y el nivel de confianza del $95 \%$.

\subsection{Selección de las variables de medida}

En relación con las variables empleadas en nuestro estudio, se han seleccionado de acuerdo a la literatura previa sobre el tema y mediante la elección de variables ya validadas en investigaciones anteriores.

- Calidad percibida. Se empleó una escala que trata de recoger el juicio global del consumidor acerca de la información o bien su superioridad frente a otros medios. La escala utilizada se basó en los trabajos de Medina y Ojer (2009) y de Gutierrez-Cea (2000).

- Conciencia social de los diarios. Se midió mediante los ítems propuestos por Gaziano y McGrath (1986).

- Notoriedad e imagen. Se emplearon los items propuestos por Boulding (1969) y por Aaker (1991), referidos al conocimiento y familiaridad que el consumidor tiene con el medio. La variable credibilidad se midió mediante items propuestos por Gaziano y McGrath (1986), así como por Newell y Goldsmith (2001).

- Actitud de los consumidores. Se midió a través de la escala planteada por Bruner y Hensel (1992).

- Fidelidad hacia el medio. Se utilizaron ítems propuestos por Nguyen y Leblanc (2001).

- Aceptación de nuevos productos. Se midió a partir de la escala propuesta por Klink y Smith (2001).

\section{Discusión de resultados}

\subsection{Análisis del modelo de medida}

En el presente estudio se ha llevado a cabo un modelo de ecuaciones estructurales, a través del programa Amos 18.0, considerando las variables como reflectivas. Antes de analizar el modelo propuesto, examinamos su estructura multidimensional con un análisis factorial confirmatorio, con el propósito de comprobar la fiabilidad y validez de la escala de medida. Un primer análisis reveló la necesidad de eliminar un ítem de la escala inicial propuesta, en concreto el relacionado con la variable imagen/notoriedad (Aso3). Una vez suprimido este indicador, los resultados muestran una especificación adecuada de la estructura factorial propuesta (tabla 1). Para el análisis de la fiabilidad y de la consistencia interna del modelo, se calcularon los valores alpha de Cronbach, los índices de fiabilidad compuesta y los valores de la varianza extraída. Siguiendo a Anderson y Gerbing (1988) y Hair et al. (1992), la fiabilidad se considera aceptable cuando los valores alfa de Cronbach superan el valor de 0,7 , valor superado por todos los constructos en nuestro análisis. En cuanto a la fiabilidad compuesta -medida de consistencia interna de los constructos-, se confirmaría en nuestro modelo, dado que los valores de los coeficientes lambda estandarizados son superiores a 0,5 (Bagozzi; Yi, 1988), a excepción del ítem Cal4. En relación con la validez discriminante, los valores de la varianza extraída también se consideran aceptables, dado que superan el valor 0,5 (Hair et al., 1999).

Para el ajuste estructural del modelo, se analizaron una serie de medidas. De acuerdo con los resultados, chi-cuadrado obtiene un valor de 871,523 (DF=386, $\quad p<0,001$ ), y se logra un índice de bondad del ajuste (GFI) de 0,878, pudiendo considerarse indicadores fiables del ajuste del modelo (Bollen, 1989; Hair et al. 1999). Asimismo, el índice de ajuste normalizado (NFI), alcanza un valor de 0,927 , siendo los valores superiores a 0,9 generalmente aceptados como evidencia de un buen ajuste (Browne; Cudeck, 1993). EI RMSEA (root mean square error of approximation) alcanza un valor de 0,053, evidenciando un ajuste aceptable del modelo general (Bagozzi; Yi, 1988). 
Las medidas del ajuste incremental, así como las medidas de parsimonia también indican un buen ajuste, dado que las primeras alcanzan valores superiores a 0,9 o bien muy próximas, como es el caso del índice de bondad del ajuste normalizado o AGFI (Hair et al., 1999). Por último, las medidas de parsimonia también indican un ajuste apropiado del modelo, dado que el índice de ajuste comparativo (CFI), el índice de ajuste normalizado (IFI) y el índice de Tucker-Lewis (TLI) alcanzan valores superiores al 0,9. (Hair et al. 1999).

\subsection{Análisis del modelo propuesto}

Nuestros resultados ponen de relieve que todas las relaciones causales planteadas son positivas y estadísticamente significativas, a excepción de la influencia de la variable conciencia social sobre la actitud, dado que no se halla suficiente evidencia empírica para proponer una relación significativa entre ambas (figura 2). El análisis de los coeficientes estandarizados muestra que los antecedentes de la credibilidad del medio de comunicación, esto es, la calidad percibida $\left(\beta_{14}=0,491\right)$, y la imagen y notoriedad $\left(\beta_{34}=0,661\right)$, tienen una influencia positiva y significativa sobre la actitud, mostrando el mayor peso. Es decir, la variable imagen y notoriedad del medio tiene un efecto dominante en la actitud del consumidor. Los resultados sugieren que la notoriedad del medio, esto es, la familiaridad de éste para el consumidor y su capacidad para reconocerlo y recordarlo, así como una imagen positiva y favorable ejercen la mayor influencia en la actitud del consumidor de información.

La variable imagen y notoriedad del medio tiene un efecto dominante en la actitud del consumidor

Sin embargo, las relaciones causales descritas en este modelo no muestran evidencia significativa de la influencia de la variable conciencia social sobre la actitud del consumidor. Esto puede deberse a que en el entorno actual, caracterizado por una crisis institucional y económica, el conocimiento de numerosos casos de corrupción se debe a la labor de los medios de comunicación -entre ellos los diarios-, y por tanto el consumidor presupone y da por supuesta dicha conciencia social de la prensa. Y es que según autores como Gaziano y McGrath (1986), la conciencia social incluye factores como el comportamiento ético y moral en el tratamiento de la información y el compromiso con el interés público, valores que se ponen de relieve en el contexto actual.

Por otro lado el análisis de la variable actitud revela su influencia positiva y significativa sobre la credibilidad $\left(\beta_{45}=0,865\right)$, la fidelidad hacia el medio $\left(\beta_{46}=0,941\right)$ y la aceptación de nuevos productos $\left(\beta_{47}=0,889\right)$. De hecho, la relación entre la actitud y la fidelidad hacia el
Tabla 2. Índices de ajuste del modelo estructural

\begin{tabular}{|l|c|}
\hline \multicolumn{2}{|c|}{ Medidas absolutas de ajuste } \\
\hline Chi-cuadrado & 871,523 \\
\hline Grados de libertad & 386 \\
\hline Nivel de significación & 0,000 \\
\hline Indice de bondad del ajuste (GFI) & 0,878 \\
\hline Root mean square error of approx (RMSEA) & 0,053 \\
\hline \multicolumn{2}{|c|}{ Medidas de ajuste incremental } \\
\hline Indice de bondad del ajuste normalizado (AGFI) & 0,853 \\
\hline Índice de ajuste normalizado (NFI) & 0,927 \\
\hline Indice del ajuste incremental (IFI) & 0,958 \\
\hline Índice de Tucker-Lewis (TLI) & 0,953 \\
\hline Indice de ajuste comparativo (CFI) & 0,958 \\
\hline \multicolumn{2}{|c|}{ Medidas de parsimonia } \\
\hline Chi cuadrado normalizada & 2,258 \\
\hline
\end{tabular}

medio es la más fuerte identificada en el modelo, poniendo de relieve la fuerte influencia que una mejora o cambio en la actitud puede ocasionar. Asimismo, la credibilidad también muestra una influencia positiva sobre la fidelidad hacia el medio $\left(\beta_{56}=0,527\right)$ y la aceptación de nuevos productos $\left(\beta_{57}=0,464\right)$, pero con un peso menor. Los resultados obtenidos están en la línea de investigaciones previas (Vila-López; Küster; Bigné, 2013) que señalan que la fidelidad hacia los diarios y la aceptación de productos nuevos se explica tanto por la actitud del consumidor, como por la credibilidad del medio.

El análisis del modelo propuesto sugiere que todas las hipótesis planteadas inicialmente se aceptan $-H_{0}, H_{2}, H_{3}, H_{4}, H_{5}$, $H_{6}$, y $H_{7}-$, a excepción de $H_{1}$. Los resultados muestran que si un diario mejora su calidad percibida o su notoriedad e imagen, ello repercutirá en una mejor actitud de los consumidores $\left(H_{0}\right.$ y $\left.H_{2}\right)$. Además, una mejora en la actitud hacia el diario conllevará una mayor fidelidad hacia el mismo $\left(H_{4}\right)$, y una mejor predisposición hacia los nuevos formatos y productos que pueda introducir en el mercado $\left(H_{5}\right)$; así como una mayor credibilidad $\left(H_{3}\right)$. Finalmente, la credibilidad del medio también influye sobre ambas consecuencias, aceptándose igualmente $H_{6}$ y $H_{7}$. Sin embargo, la hipótesis inicial $H_{1}$ : La conciencia social del diario tiene una influencia positiva en la actitud hacia el mismo, no puede ser aceptada, dado que no se halla suficiente evidencia empírica para proponer una relación significativa entre ambas variables.

Tabla 3. Relaciones causales entre variables y contraste de hipótesis (coeficientes estandarizados)

\begin{tabular}{|c|c|c|c|}
\hline Relaciones causales & $\begin{array}{c}\text { Coeficientes } \\
\text { estandarizados }\end{array}$ & Valor t & Hipótesis \\
\hline Calidad percibida $\longrightarrow$ Actitud & $\beta_{14=} 0,491^{*}$ & 2,125 & $H_{0}=$ Se acepta \\
\hline Conciencia social $\longrightarrow$ Actitud & $\beta_{24=0,084^{\mathrm{ns}}}$ & 0,508 & $H_{1}=$ No se acepta \\
\hline Imagen/notoriedad $\longrightarrow$ Actitud & $\beta_{34=0,661^{*}}$ & 3,594 & $\mathrm{H}_{2}=$ Se acepta \\
\hline Actitud $\longrightarrow$ Credibilidad & $\beta_{45=0,865^{*}}$ & 6,741 & $\mathrm{H}_{3}=$ Se acepta \\
\hline Actitud $\longrightarrow$ Fidelidad & $\beta_{46=} 0,941^{*}$ & 6,856 & $H_{4}=$ Se acepta \\
\hline Actitud $\longrightarrow$ Aceptación nuevos productos & $\beta_{47=0,889^{*}}$ & 6,820 & $H_{5}=$ Se acepta \\
\hline Credibilidad $\longrightarrow$ Fidelidad & $\beta_{56=0,527^{*}}$ & 2,719 & $H_{6}=$ Se acepta \\
\hline Credibilidad $\longrightarrow$ Aceptación nuevos productos & $\beta_{57=0,464^{*}}$ & 1,967 & $H_{7}=$ Se acepta \\
\hline $\begin{array}{l}X^{2}=871,523 \quad d f=386 \quad p=, 000 \\
R^{2}(\text { Actitud })=0,883 \quad R^{2}(\text { Credibilidad })=0,932 \\
n s=\text { no significativo } \\
\text { * significativo }(p<0,05)\end{array}$ & & & \\
\hline
\end{tabular}




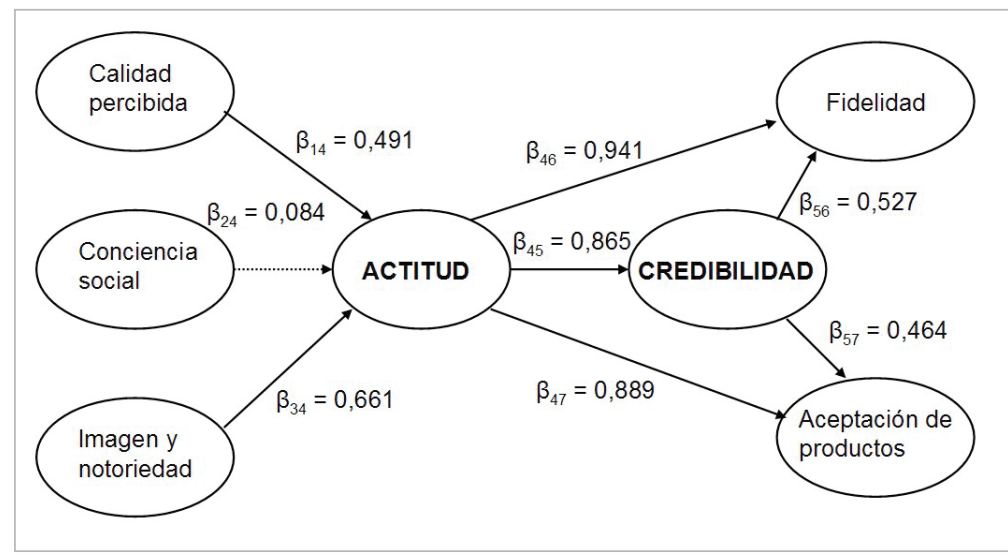

Figura 2. Relaciones causales

\section{Conclusiones}

En primer lugar, los resultados obtenidos señalan el carácter multidimensional del concepto de credibilidad de los medios de comunicación (Gaziano; McGrath, 1986), que se prueba empíricamente para el caso de los diarios generalistas y de referencia.

En segundo lugar, se confirma la existencia de un orden causal en el proceso de formación de la credibilidad del medio de comunicación, y el efecto positivo de dicha credibilidad en el comportamiento del consumidor, más concretamente en su fidelidad hacia el medio y su predisposición a aceptar nuevos formatos y productos.

\section{Si un diario mejora su calidad percibida o su notoriedad e imagen, ello repercu- tirá en una mejor actitud de los consu- midores}

En cuanto a los antecedentes de la credibilidad, se confirma la existencia de una relación positiva y significativa entre la calidad percibida, la notoriedad e imagen y la actitud del consumidor de información. De manera que cuanto mayor sea la notoriedad del diario, su calidad percibida y más favorable sea su imagen de la marca, más favorable será la actitud de los consumidores y mejor será su valoración. Por tanto, para la construcción de un medio creíble, se debe tener en cuenta la calidad que el consumidor infiere a partir de su experiencia (Gotsi; Wilson, 2001). Asimismo, la creación de un medio con una imagen positiva, favorable y familiar entre los consumidores es uno de los primeros pasos que las empresas de la industria de la comunicación deben tener en cuenta para la creación de un medio creíble, dado que una imagen e identidad sólida contribuyen notablemente a incrementar la credibilidad. Esto es, una imagen atractiva y sólida genera la percepción de que el medio es creíble.

De la misma manera, se muestra la existencia de una relación positiva entre la actitud y la credibilidad del medio, así como de ambas variables sobre la fidelidad y la aceptación hacia nuevos productos por parte de los consumidores. Podemos concluir que la creación y el incremento de la fidelidad de los consumidores es especialmente importante para los medios de comunicación en un entorno de gran competencia y fragmentación del sector; y por lo tanto, los gestores de los medios han de buscar y procurar una audiencia o un consumidor fiel, siendo crucial la credibilidad percibida del medio. Se puede afirmar que la creación de medios de comunicación creíbles se convierte en una prioridad para las empresas.

Nuestra investigación añade valor a la literatura existente, mediante una comprensión más profunda del proceso de creación de credibilidad de los medios de comunicación, para el caso de los diarios generalistas y de referencia, mostrando las relaciones entre las variables antecedentes, así como en las consecuencias que tiene en la respuesta por parte del consumidor de información.

A la luz de estos resultados cabe señalar una serie de implicaciones para los responsables de los medios de comunicación.

- Sugerimos una apuesta clara por incrementar y mejorar la credibilidad del medio, especialmente en un entorno tan competitivo, lo que hace necesario que los directivos de los medios analicen y conozcan la credibilidad que transmiten (Balmer; Greyser, 2003), entendiendo la credibilidad como un concepto multidimensional que recoge atributos como honestidad, habilidad, experiencia y confianza asociados al medio.

- Para conseguir la credibilidad del medio es fundamental mejorar la calidad percibida del mismo, así como reforzar e incrementar su notoriedad e imagen. La mejora de la calidad percibida debería pasar por ofrecer un contenido tanto de información como de opinión, dirigido a una audiencia heterogénea, con pluralidad de creencias y opiniones, proporcionando información de calidad, sin sesgos, contando la historia completa y contribuyendo a la formación de opinión pública (Ortega, 2008; BignéAlcañiz; Currás-Pérez; Sánchez-García, 2009).

- Los gestores deben mejorar la notoriedad e imagen de los diarios, comenzando con su identidad visual, actualizando su logotipo, tipografía, nombres y símbolos (Van-Weezel, 2006). Para ello las empresas periodísticas han de destinar recursos para el incremento y mejora de su notoriedad en el mercado, su familiaridad y capacidad de reconocimiento y recuerdo por parte de los consumidores; así como para crear una cabecera visible y diferenciada de otros diarios, mediante el empleo de una marca y un logotipo claramente identificables. En definitiva los diarios deben proveer valor a los consumidores de información, invitándoles a una experiencia placentera a través de sus contenidos (Erdem; Swait, 1998).

En cuanto a las principales limitaciones del estudio, se debe señalar lo siguiente:

- la investigación empírica se ha circunscrito a España, así como a un tipo específico de medio de comunicación -la prensa escrita generalista y de referencia-, lo que plantea serias dificultades a la hora de extrapolar los resultados al conjunto de medios de comunicación presentes en el 
mercado, ya sean tradicionales o en soporte digital;

- nuestro trabajo ha analizado una serie de variables propuestas anteriormente por la literatura, obviando otras importantes en el estudio de la credibilidad o actitud hacia los medios, como la familiaridad con el medio (VilaLópez; Küster; Bigné, 2013) o la reputación o prestigio (Newell; Goldsmith, 2001; Herrera-Damas, 2013). Por estos motivos, las relaciones que pone de manifiesto nuestra investigación deben ser tomadas con cautela.

Los gestores de los medios han de buscar y procurar una audiencia o un consumidor fiel, siendo crucial la credibilidad percibida del medio

\section{Bibliografía}

Aaker, David A. (1991). Managing brand equity capitalizing on the value of brand name. New York: The Free Press. ISBN: 0029001013

Amos, Clinton; Holmes, Gary; Strutton, David (2008). “Exploring the relationship between celebrity endorser effects and advertising effectiveness: a quantitative synthesis of effect size". International journal of advertising, v. 27, n. 2, pp. 209-234, ISSN: 02650487

http://hull.aug.edu/hcbWeb/media/research/AmosHolmes-Strutton-IJA-2008.pdf

Anderson, James C.; Gerbing, David W. (1988). "Structural equation modeling in practice: A review and recommended two step approach". Psychological bulletin, v. 103, pp. 411423.

http://www.fdewb.unimaas.nl/meteor/EDEN/Mike\%20 Brady/Anderson\%20and\%20Gerbing\%201988.pdf

Bagozzi, Richard P.; Yi, Youjae (1988). “On the evaluation of structural equation models". Journal of the Academy of Marketing Science, v. 16, n. 1, pp. 74-94. http://dx.doi.org/10.1007\%2FBF02723327

Balmer, John; Greyser, Stephen (2003). Revealing the corporation. Perspectives on identity image, reputation, corporate branding and corporate-level marketing. Routledge: London. ISBN: 0415284201

Bollen, Kenneth (1989). Structural equations with latent variables. Wiley series in probability and mathematical statistic. John Wiley and Sons, New York. ISBN: 0471011711

Bigné-Alcañiz, José-Enrique (1992). "El efecto persuasivo de la fuente: su extensión objetiva”. Alta dirección, v. 27, n. 162, marzo-abril, pp. 75-83.

Bigné-Alcañiz, José-Enrique; Currás-Pérez, Rafael; SánchezGarcía, Isabel (2009). "Brand credibility in cause-related marketing: the moderating role of consumer values". Journal of product and brand management, v. 18, n. 6, pp. 437 447.

http://dx.doi.org/10.1108/10610420910989758

Blackwell, Roger; Engel, James; Miniard, Paul (2002). Com- portamiento del consumidor. México: Thompson. ISBN: 970 6861874

Boulding, Kenneth E. (1969). The image: knowledge in life and society. 7th edition. Michigan: Ann Arbor, The University of Michigan Press. ISBN: 0472060473

Browne, Michael W.; Cudeck, Robert (1993). Alternative ways of assessing model fit. In: Bollen, Kenneth A.; Long, J. Scott (eds.). Testing structural equation models. Sage Publications. Thousand Oaks, C. A. pp. 136-161. ISBN: 08039 $4506 \mathrm{X}$

Bruner, Gordon; Hensel, Paul (1992). Marketing scales handbook: a compilation of multi item measures. Chicago: American Marketing Association. ISBN: 0877572267

Carrillo, María-Victoria; Castillo-Díaz, Ana; Tato-Jiménez, Juan-Luis (2008). "El valor de lo intangible: La gestión de la reputación corporativa. El caso de la marca Telefónica". Observatorio journal, v. 7, pp. 239-254.

http://obs.obercom.pt/index.php/obs/article/ download/123/215

Chan-OImsted, Sylvia M.; Yungwook, Kim (2001). "Perceptions of branding among television station managers: an exploratory study". Journal of broadcasting \& electronic media, v. 45, n. 1, pp. 75-91. http://dx.doi.org/10.1207\%2Fs15506878jobem4501_6

Dick, Alan; Basu, Kunal (1994). "Customer loyalty: towards an integrated framework". Journal of the academy of marketing science, v. 22, n. 2, pp. 99-113. http://dx.doi.org/10.1177/0092070394222001

Erdem, Tülin; Swait, Joffre (1998). "Brand equity as a signalling phenomenon". Journal of consumer psychology, v. 7, n. 2, pp. 131-157.

http://markenmanagement.files.wordpress.com/2012/01/ erdem_brandequityasasignalinphenomenon.pdf http://dx.doi.org/10.1207\%2Fs15327663jcp0702_02

Erdem, Tülin; Swait, Joffre (2004). "Brand credibility, brand consideration and choice". Journal of consumer research, $v$. 31, n. 1, pp. 191-198. http://dx.doi.org/10.1086\%2F383434

Flanagin, Andrew J.; Metzger, Miriam J. (2000). "Perceptions of internet information credibility". Journalism and mass communication quarterly, v. 77, n.3, pp. 515-540. http://www.jasonmorrison.net/iakm/4006074.pdf http://dx.doi.org/10.1177\%2F107769900007700304

Fogg, Brian J. (2003). "Prominence-interpretation theory: explaining how people assess credibility online". En: Conference on Human Factors in Computing Systems, pp. $722-$ 723. Ft. Lauderdale: Florida (USA), ISBN: 1581136374 http://credibility.stanford.edu/pdf/PITheory.pdf http://dx.doi.org/10.1145\%2F765948.765951

Fombrun, Charles J.; Van-Riel, Cees (2004). Fame and fortune: how successful companies build winning reputations. Financial Times Prentice-Hall: Upper Saddle River, NJ. ISBN: 0130937371

Gaziano, Cecile; McGrath, Kristin (1986). "Measuring the concept of credibility". Journalism quarterly, autumn, pp. 
451-462.

http://www.aejmc.org/home/wp-content/uploads/2012/09/

Journalism-Quarterly-1986-Gaziano-451-621.pdf

http://dx.doi.org/10.1177\%2F107769908606300301

Goldsmith, Ronald; Lafferty, Barbara; Newell, Stephen (2000). "The influence of corporate credibility on consumer attitudes and purchase intent". Corporate reputation review, v. 3, n. 4, pp. 304-318.

http://dx.doi.org/10.1057\%2Fpalgrave.crr.1540122

Gotsi, Manto; Wilson, Alan (2001). “Corporate reputation: seeking a definition". Corporate communications: an international journal, v. 6, n. 1, pp. 24-30.

http://dx.doi.org/10.1108/13563280110381189

Greer, Jennifer (2003). "Evaluating the credibility of online information: A test of source and advertising influence". Mass communication and society, v. 6, n. 11, pp. 11-28. http://dx.doi.org/10.1207\%2FS15327825MCS0601_3

Gutiérrez-Cea, Charo (2000). "Televisión y calidad: perspectivas de investigación y criterios de evaluación". Revista de estudios de comunicación, v. 9, pp. 1-20.

Haley, Eric (1996). "Exploring the construct of organization as source: consumers' understandings of organizational sponsorship of advocacy advertising". Journal of advertising, v. 25, n. 2, pp. 19-3.

http://dx.doi.org/10.1080/00913367.1996.10673497

Hair, Joseph F.; Anderson, Rolph E.; Tatham, Ronald L.; Black, William (1992). Multivariable data analysis. 5th ed. Prentice Hall, Upper Saddle River, New Jersey. ISBN: 013 8948585

Hair, Joseph F.; Anderson, Rolph E.; Tatham, Ronald L.; Black, William (1999). Análisis Multivariante. Prentice Hall, Madrid, ISBN: 8483220350

Herrera-Damas, Susana (2013). "Indicaciones recurrentes en las normativas para el uso periodístico de las redes sociales". El profesional de la información, enero-febrero, v. 22, n. 1, pp. 46-53.

http://dx.doi.org/10.3145/epi.2013.ene.06

Hovland, Carl; Janis, Irving; Kelley, Harold (1953). Communication and persuasion. Psychological studies of opinion change. Yale University Press, New Haven. ISBN: 030 0005733

Klink, Richard R.; Smith, Daniel C. (2001). "Threats to the external validity of brand extension research". Journal of marketing research, v. 38, pp. 326-35.

http://dx.doi.org/10.1509\%2Fjmkr.38.3.326.18864

Lafferty, Barbara; Goldsmith, Ronald (2004). “How influential are corporate credibility and endorser attractiveness when innovations react to advertisements for a new hightechnology product". Corporate reputation review, n. 7, n. 1, pp. 24-36.

http://dx.doi.org/10.1057\%2Fpalgrave.crr.1540209

Liu, Ziming (2004). "Perceptions of credibility of scholarly information on the web". Information processing and management, v. 40, n. 6, pp. 1027-1038.

http://dx.doi.org/10.1016/S0306-4573(03)00064-5
Medina, Mercedes; Ojer, Teresa (2009). “Valoración del servicio público de televisión: comparación entre la BBC y TVE". Revista latina de comunicación social, v. 64, pp. 275-299. http://dx.doi.org/10.4185/RLCS-64-2009-823-275-299

Metzger, Miriam J.; Flanagin, Andrew J.; Eyal, Karen; Lemus, Daisy R.; McCann, Robert M. (2003). "Credibility for the 21st century: integrating perspectives on source, message and media credibility in the contemporary media environment". Communication yearbook, v. 27, pp. 293-336. http://dx.doi.org/10.1207/s15567419cy2701_10

Meyer, Philip (1998). "Imagining public journalism". En: Lambeth, Edmund; Meyer, Philip; Thorson, Esther (Eds.). Assessing public journalism. Columbia: University of Missouri Press, pp. 251-273. ISBN: 0826211585

Meyer, Philip (2004). The vanishing newspaper: saving journalism in the information age. Columbia: University of Missouri Press. ISBN: 0826218776

Mueller, Eva (1957). "Effects of consumer attitudes on purchases". The American economic review, pp. 946-965.

Newell, Stephen J.; Goldsmith, Ronald E. (2001). "The development of a scale to measure perceived corporate credibility". Journal of business research, v. 52, n. 3, pp. 235-247. http://dx.doi.org/10.1016\%2FS0148-2963\%2899\%2900104-6

Nguyen, Nha; Leblanc, Gaston (2001). “Corporate image and corporate reputation in customers retention decisions in services". Journal of retailing and consumer services, v. 8 , n.4, pp. 227-236.

http://dx.doi.org/10.1016\%2FS0969-6989\%2800\%2900029-1

Nozato, Yoshiko (2002). "Credibility of online newspapers". Annual Convention of Association for Education in Journalism and Mass Communication. Washington D.C., August.

Ortega, Félix (2008). "La encrucijada de la televisión pública en Chile". Cuadernos de información, v. 23, pp. 42-61. http://cuadernos.uc.cl/uc/index.php/CDI/article/ view/95/87

Piñeros-Piza, Catalina; Gutiérrez-Coba, Liliana-María; Gómez-Díaz, Javier-Andrés; Pérez-Acosta, Andrés M.; Salgado-Cardona, Andrea; Mora, María-Lucía; Grijalva, Nataly; Estrada, Juliana; Ramírez, María-José (2011). “Credibilidad percibida del periodismo a través de la Internet: una visión desde la psicología del consumidor". Perspectivas psicológicas, v. 7, n. 2, pp. 225-238.

http://www.usta.edu.co/otraspaginas/diversitas/doc_pdf/ diversitas_11/vol.7no.2/02_credibilidad_percibida.pdf

Pornpitakpan, Chanthika (2004), "The persuasiveness of source credibility: a critical review of five decades's evidence". Journal of applied social psychology, v. 34, n. 2, pp. 243281.

http://dx.doi.org/10.1111/j.1559-1816.2004.tb02547.x

Rathnayake, Chamil-Viranga (2008). "Brand personality and its impact on brand feelings: a study among young television viewers". South Asian journal of management, v. 15, n. 2, pp. 7-26, ISSN: 09715428

Rieh, Soo-Young; Danielson, David (2007). "Credibility: a multidisciplinary framework". Annual review of information 
science and technology, v. 41, n. 1, pp. 307-364.

http://rieh.people.si.umich.edu/ rieh/papers/rieh_ ARIST2007.pdf

http://dx.doi.org/10.1002\%2Faris.2007.1440410114

Rust, Roland T.; Donthu, Naveen (1988). “A programming and positioning strategy for cable television". Journal of advertising, v. 17, n. 4, pp. 6-13.

http://dx.doi.org/10.1080\%2F00913367.1988.10673124

Schweiger, Wolfang (2000). "Media credibility: experience or image?". European journal of communication, v. 15, n. 37, pp. 37-59.

http://dx.doi.org/10.1177/0267323100015001002

Sughan, Steven (1980). "The cost of thinking". Journal of consumer research, v. 7, pp. 99-111.

Taylor, Robert-Saxton (1986). Value-added processes in information systems. Norwood, NJ: Ablex. ISBN: 978 0893912734

Trimble, Carrie; Rifon, Nora (2006). “Consumer perceptions of compatibility in cause-related marketing messages". International journal of nonprofit and voluntary sector marketing, v. 11, n. 1, pp. 29-47.

http://dx.doi.org/10.1002\%2Fnvsm.42

Tseng, Shawn; Fogg, B. J. (1999). “Credibility and computing technology". Communications of the ACM, v. 42, n. 5, pp. 39-44.

http://ww.mehmetgokturk.com/files/hcicourse/p39-tseng. $p d f$

http://dx.doi.org/10.1145\%2F301353.301402

Van-Weezel, Aldo (2006). "Contenidos de calidad: una apuesta lógica". Cuadernos de gestión, v. 19, pp. 62-67. http://www.redalyc.org/articulo.oa?id=97117399009

Vila-López, Natalia; Küster-Boluda, Inés; Bigné, Enrique
(2013). “Credibilidad corporativa en cadenas televisivas: antecedentes y efectos". Revista española de investigación de marketing ESIC, v. 17, n. 1, pp. 61-82.

http://www.esic.edu/documentos/revistas/ reim/130320_161859_E.pdf

Walsh, Gianfranco; Mitchell, Vincent; Jackson, Paul; Beatty, Sharon (2009). "Examining the antecedents and consequences of corporate reputation: a customer perspective". British journal of management, v. 20, n. 2, pp. 187-203. http://dx.doi.org/10.1111\%2Fj.1467-8551.2007.00557.x

Wang, Peiling; Soergel, Dagobert (1998). "A cognitive model of document use during a research project Study 1: Document selection". Journal of the American Society for Information Science, v. 49, n. 2, pp. 115-133.

http://www.asis.org/Publications/JASIS/Best_ Jasist/1999WangandSoergel.pdf

http://dx.doi.org/10.1002/(SICI)1097-4571(199802)49:2<115::AIDASI3>3.0.CO;2-T

Wathen, Nadine; Burkell, Jacquelyn (2002). "Believe it or not, factors influencing credibility on the web". Journal of the American Society for Information Science and Technology, v. 53, n. 2, pp. 134-144.

http://dx.doi.org/10.1002/asi.10016

Webster, James G. (1986) "Audience behavior in the new media environment". Journal of communication, v. 36, n. 6, pp. 77-94.

http://dx.doi.org/10.1111/j.1460-2466.1986.tb01439.x

Woo, Jong; Cho, Chang-Hoan; Kwon, Joon, (2008). "The role of affect and cognition in consumer evaluations of corporate visual identity: Perspectives from the United States and Korea". Journal of brand management, v. 15, n.6, pp. 382-398

http://dx.doi.org/10.1057/bm.2008.11

\section{Próximos temas centrales}

Julio 2014

Septiembre 2014

Noviembre 2014

Enero 2015

Marzo 2015

Mayo 2015

Julio 2015

\section{Altmétricas}

Humanidades digitales

Big data y analítica web

Marketing de productos de información

Web semántica

Privacidad y seguridad de información

Bases de datos bibliográficas

Los interesados por favor consulten detenidamente las Normas para autores:

http://www.elprofesionaldelainformacion.com/autores.html

y luego envíen sus artículos a través del gestor de manuscritos OJS de la plataforma del Recyt: http://recyt.fecyt.es/index.php/EPI/index 